\title{
$A b$ initio derivation and exact diagonalization analysis of low-energy effective Hamiltonians for $\beta^{\prime}-\mathrm{X}\left[\mathrm{Pd}(\mathrm{dmit})_{2}\right]_{2}$
}

\author{
Kazuyoshi Yoshimi $\odot,{ }^{1}$ Takao Tsumuraya $\odot,{ }^{2}$ and Takahiro Misawa $\odot^{3}$ \\ ${ }^{1}$ Institute for Solid State Physics, University of Tokyo, 5-1-5 Kashiwanoha, Kashiwa, Chiba 277-8581, Japan \\ ${ }^{2}$ Priority Organization for Innovation and Excellence, Kumamoto University, 2-39-1 Kurokami, Kumamoto 860-8555, Japan \\ ${ }^{3}$ Beijing Academy of Quantum Information Sciences, Haidian District, Beijing 100193, China
}

(Received 22 September 2021; accepted 10 December 2021; published 28 December 2021)

\begin{abstract}
The molecular solids $\beta^{\prime}-\mathrm{X}\left[\mathrm{Pd}(\mathrm{dmit})_{2}\right]_{2}$ (where $\mathrm{X}$ represents a cation) are typical compounds whose electronic structures are described by single-orbital Hubbard-type Hamiltonians with geometrical frustration. Using the $a b$ initio downfolding method, we derive the low-energy effective Hamiltonians for $\beta^{\prime}-\mathrm{X}\left[\mathrm{Pd}(\mathrm{dmit})_{2}\right]_{2}$ with available room- and low-temperature structures. We find that the amplitudes of the Coulomb interactions and the anisotropy of the hopping parameters in the effective Hamiltonians are sensitive to the changes in the lattice constants induced by lowering the temperature. The obtained effective Hamiltonians are analyzed using the exact diagonalization method with the boundary condition average. We find that a significant reduction of the antiferromagnetic ordered moment occurs in the effective Hamiltonian of $\beta^{\prime}-\mathrm{EtMe}_{3} \mathrm{Sb}\left[\mathrm{Pd}(\mathrm{dmit})_{2}\right]_{2}$ with the low-temperature structure. The reduction is consistent with the quantum spin liquid behavior observed in experiments. The comprehensive derivations of the effective Hamiltonians and exact-diagonalization analyses of them will clarify the microscopic origins of the exotic quantum states of matter found in $\beta^{\prime}-\mathrm{X}\left[\mathrm{Pd}(\mathrm{dmit})_{2}\right]_{2}$ such as the quantum spin liquid behavior.
\end{abstract}

DOI: 10.1103/PhysRevResearch.3.043224

\section{INTRODUCTION}

The single-orbital Hubbard model and its extensions have been studied as canonical models that show exotic quantum phases of matter such as high- $T_{\mathrm{c}}$ superconducting phases [1] and quantum spin liquids (QSLs) [2]. It has been shown that some families of molecular solids such as (BEDT-TTF $)_{2} X$ with $\kappa$ and $\beta^{\prime}$-type dimer arrangements offer an ideal platform for realizing the single-orbital Hubbard model [3-5]. Although most molecular solids have a complex structure typically containing more than 100 atoms in the unit cell, the low-energy band structures near the Fermi level are simple, i.e., one frontier molecular orbital of the constituent molecule contributes to the formation of the conduction bands and the other orbitals are sufficiently far from the Fermi level [6]. In fact, the simple band structures near the Fermi level have been confirmed by several experiments, such as de Haas-van Alphen (dHvA) measurements [7]. The simple isolated band structures in the molecular solids are in sharp contrast with those of transition metal oxides such as cuprates, where $p$ orbitals in oxygen often largely hybridize with $d$ orbitals in transition metals $[8,9]$.

Among molecular solids, the anion radical salt of $\beta^{\prime}-\mathrm{X}\left[\mathrm{Pd}(\mathrm{dmit})_{2}\right]_{2} \quad(\mathrm{dmit}=1,3$-dithiole-2-thione-4,5-

Published by the American Physical Society under the terms of the Creative Commons Attribution 4.0 International license. Further distribution of this work must maintain attribution to the author(s) and the published article's title, journal citation, and DOI. dithiolate; $\mathrm{X}=$ a monovalent closed-shell cation) is a typical compound whose electronic structures can be described by the single-orbital Hubbard-type Hamiltonian. In these solids, the $\left[\operatorname{Pd}(\mathrm{dmit})_{2}\right]$ unit is strongly dimerized and the dimers form an anisotropic triangular lattice in each anion layer. In the dimer unit, both the HOMO and LUMO of the Pd(dmit $)_{2}$ monomer form bonding and antibonding pairs, and a half-filled band is mainly formed through the antibonding HOMO pair [10-13]. Experimentally, by changing the type of cation $\mathrm{X}$, a rich variety of ground states such as antiferromagnetically (AF) ordered states [14], QSL states [15-17], and charge ordered (CO) states $[18,19]$ have been found.

In our previous study [20], we have shown that the single-orbital extended Hubbard-type Hamiltonians obtained in $a b$ initio calculations successfully reproduce the cation dependence of the magnetic properties in the dmit salts, i.e., the peak value of the spin structure factors is significantly suppressed around the QSL compound $\mathrm{X}=\mathrm{EtMe}_{3} \mathrm{Sb}$ salt (Et $=\mathrm{C}_{2} \mathrm{H}_{5}$ and $\mathrm{Me}=\mathrm{CH}_{3}$, hereafter called the $\mathrm{X}$ salt, for simplicity). We found that the magnetic moment of $\mathrm{EtMe}_{3} \mathrm{Sb}$ salt reduces only in the effective Hamiltonian of the lowtemperature (LT) structure of $4 \mathrm{~K}$ and not in that of the room temperature (RT) structure. This result indicates that changes in a lattice structure (i.e., the distance between dimers) due to a decrease in temperature significantly influence the electronic structures of the dmit salts.

To date, changes in the lattice structures for decreasing temperatures have not been seriously examined in the context of ab initio derivations of the low-energy effective Hamiltonians. Most studies of $a b$ initio derivations of the low-energy effective Hamiltonians have employed the experimental 
structure at RT to avoid the effects of symmetry breaking at LT, such as magnetic orderings [21-26]. This treatment is justified when the changes in the lattice constants are small. However, molecular solids are flexible because they are loosely bounded by van der Waals interactions. Thus it is expected that changes in the lattice structures by lowering the temperature significantly affect the electronic structures of the dmit salts. Although it was reported that the transfer integrals between dimers for LT structures of $\mathrm{EtMe}_{3} \mathrm{Sb}(4.5 \mathrm{~K})$ [27] and $\mathrm{Me}_{4} \mathrm{P}$ salts ( $8 \mathrm{~K}$ ) [28] show a larger anisotropy of the triangular lattice than those at RT [20,24,29], the effects of lowering the temperature for other compounds has not been clarified. More recently, the LT structures of $\mathrm{Et}_{2} \mathrm{Me}_{2} \mathrm{As}$, $\mathrm{Me}_{4} \mathrm{As}$, and $\mathrm{Me}_{4} \mathrm{Sb}$ salts have been reported [30]. Using these LT structures, it is now possible to clarify how the changes in the structures affect the microscopic parameters and physical quantities in the effective Hamiltonians of the dmit salts.

In this paper, using the ab initio downfolding method [31,32], we derive low-energy effective Hamiltonians for nine dmit salts with RT structures and five dmit salts with LT structures. We clarify how the changes in the lattice constants affect the microscopic parameters in the effective Hamiltonians, such as the transfer integrals and Coulomb interactions. By solving the derived effective Hamiltonians using the exact diagonalization method, we also clarify the effects of changes in the lattice constants on the physical quantities, such as the spin structure factors and the charge gap. We find that all the Hamiltonians for the LT structures show stripe-type magnetic ordered correlations and significant reductions in the magnetic ordered moment occur around the $\mathrm{EtMe}_{3} \mathrm{Sb}$ salt. This result is consistent with the compound dependence of the Néel temperatures and the quantum spin liquid behavior observed in $\mathrm{EtMe}_{3} \mathrm{Sb}$ salt [15-17].

The remainder of this paper is organized as follows. In Sec. II, the methods to derive low-energy effective Hamiltonians and analyze them are described. In Sec. III, the values of parameters such as transfer integrals and Coulomb interactions, which constitute the effective Hamiltonians, are listed for each material and temperature, and their trends are discussed. In addition, by analyzing these models using the exact diagonalization method, we obtain the compound dependence of the spin structure factors and the charge gap. In the exact-diagonalization analyses, to reduce the finite-size effects, we performed the boundary-condition average [33]. We also discuss how our obtained results can be observed in experiments. Section IV contains a summary of the paper.

\section{METHODS}

\section{A. Derivation of low-energy effective Hamiltonians}

In this study, the low-energy effective Hamiltonians are derived from the nonmagnetic band structure obtained using first-principles density functional theory (DFT) calculations $[34,35]$. In the series of DFT calculations, we refer to the experimental structures at LT and RT $[21,30]$. The RT structures of the nine members of $\beta^{\prime}$ salts are isostructural, with a face-centered monoclinic structure of the space group $C 2 / c$. No structural phase transition is observed by lowering the temperature for all the salts except for the $\mathrm{Et}_{2} \mathrm{Me}_{2} \mathrm{Sb}$ salt, which causes CO. In this work, we study the cation dependence of the electronic state of the lowest temperature for $\mathrm{EtMe}_{3} \mathrm{Sb}(5 \mathrm{~K}), \mathrm{Me}_{4} \mathrm{P}(8 \mathrm{~K}), \mathrm{Me}_{4} \mathrm{As}(5 \mathrm{~K}), \mathrm{Me}_{4} \mathrm{Sb}(5 \mathrm{~K})$, and $\mathrm{Et}_{2} \mathrm{Me}_{2} \mathrm{As}(5 \mathrm{~K})$ salts for which the experimental structures have been reported $[28,30]$.

The preset first-principles calculations were performed using the pseudopotential method based on the optimized norm-conserving Vanderbilt (ONCV) formalism with planewave basis sets [36,37], which is implemented in QUANTUM ESPRESSO (VERSION 6.3) [38]. The exchange-correlation functional of the generalized gradient approximation (GGA) proposed by Perdew, Burke, and Ernzerhof (PBE) was employed [39]. The cutoff energies for plane waves and charge densities are 70 and $280 \mathrm{Ry}$, respectively. A $5 \times 5 \times 3$ uniform $\boldsymbol{k}$-point mesh was used for all the $\beta^{\prime}$ salts with a Gaussian smearing method during self-consistent loops. All the hydrogen positions were relaxed through the geometrical optimization procedure of the first-principles method because it is difficult to accurately determine $\mathrm{C}-\mathrm{H}$ bond distances from $\mathrm{X}$-ray diffraction measurements.

Using the obtained Bloch functions, we generated maximally localized Wannier functions (MLWF) for the half-filled bands crossing the Fermi level using RESPACK [40]. Then, the transfer integral between MLWFs at $\boldsymbol{R}_{1}$ and $\boldsymbol{R}_{2}$ sites is given as follows:

$$
t_{\boldsymbol{R}_{1} \boldsymbol{R}_{2}}=\left\langle\phi_{\boldsymbol{R}_{1}}\left|H_{0}\right| \phi_{\boldsymbol{R}_{2}}\right\rangle
$$

Here, $\left|\phi_{R}\right\rangle=c_{R}^{\dagger}|0\rangle$, where $c_{R}^{\dagger}$ is the creation operator of an electron in the Wannier orbital at $\boldsymbol{R}$ site and $H_{0}$ represents the noninteraction term of the $a b$ initio Hamiltonians. In Fig. 1, we show the band structure and the MLWFs of $\mathrm{EtMe}_{3} \mathrm{Sb}$ and $\mathrm{Me}_{4} \mathrm{P}$ salts, which we will discuss in Sec. III. The initial coordinates of the MLWF are set at the center between two $\left[\mathrm{Pd}(\mathrm{dmit})_{2}\right]$ monomers to generate a one-band model (socalled dimer model) [41].

The interactions were evaluated by the constrained random-phase approximation (cRPA) [31] method using RESPACK. The energy cutoff for the dielectric function was set to be $3 \mathrm{Ry}$. The interaction terms are given as follows:

$$
W\left(\boldsymbol{R}_{1}, \boldsymbol{R}_{2}, \boldsymbol{R}_{3}, \boldsymbol{R}_{4}\right)=\left\langle\phi_{\boldsymbol{R}_{1}} \phi_{\boldsymbol{R}_{2}}\left|H_{W}\right| \phi_{\boldsymbol{R}_{3}} \phi_{\boldsymbol{R}_{4}}\right\rangle,
$$

where $H_{W}$ represents the interaction term of the $a b$ initio Hamiltonians. We only treat the two-body interactions, such as density-density interactions $U(\boldsymbol{R})=W(\mathbf{0}, \mathbf{0}, \boldsymbol{R}, \boldsymbol{R})$ (that is, the on-site and off-site Coulomb interactions) and direct exchange interactions $J(\boldsymbol{R})=W(\mathbf{0}, \boldsymbol{R}, \boldsymbol{R}, \mathbf{0})$, because the amplitudes of the other terms are negligibly small.

From the above calculations, the following extended single-band Hubbard-type Hamiltonian is obtained:

$$
\begin{aligned}
H= & \sum_{i j, \sigma} t_{i j}\left(c_{i \sigma}^{\dagger} c_{j \sigma}+\text { H.c. }\right)+U \sum_{i} n_{i \uparrow} n_{i \downarrow}+\sum_{i j} V_{i j} N_{i} N_{j} \\
& +\sum_{i j, \sigma \rho} J_{i j}\left(c_{i \sigma}^{\dagger} c_{j \rho}^{\dagger} c_{i \rho} c_{j \sigma}+c_{i \sigma}^{\dagger} c_{i \rho}^{\dagger} c_{j \rho} c_{i \sigma}\right)
\end{aligned}
$$

where $c_{i \sigma}^{\dagger}$ and $c_{i \sigma}$ are the creation and annihilation operators of an electron with spin $\sigma$ in the Wannier orbital localized at the $i$ th dmit dimers. The number operators are defined as $n_{i \sigma}=$ $c_{i \sigma}^{\dagger} c_{i \sigma}$ and $N_{i}=n_{i \uparrow}+n_{i \downarrow}$. The obtained model parameters are 
(a)

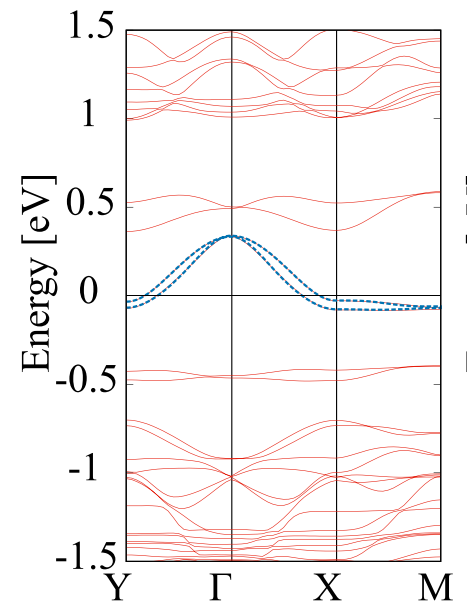

(d)

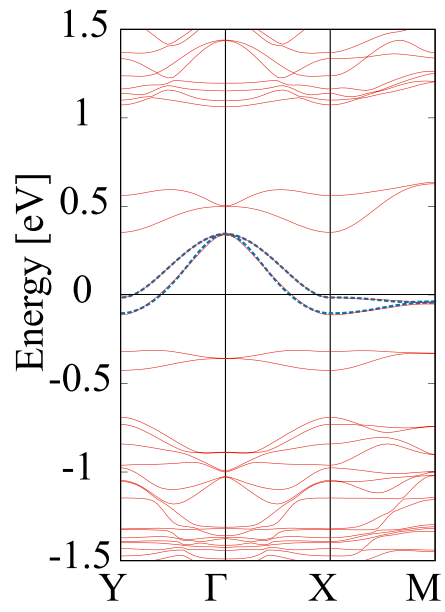

(b)

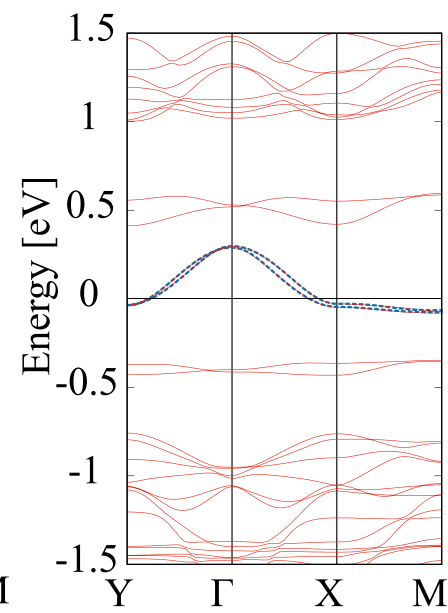

(e)

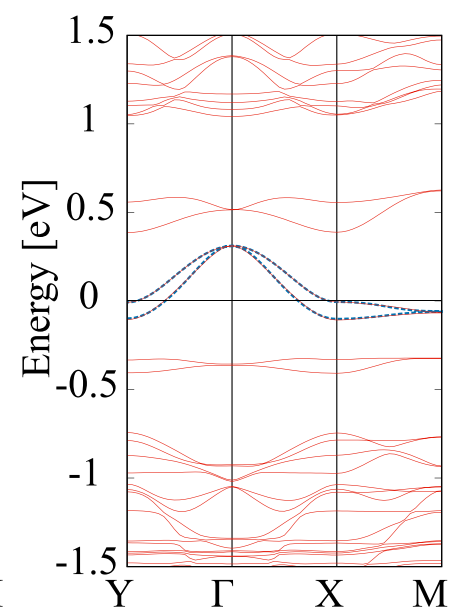

(c)

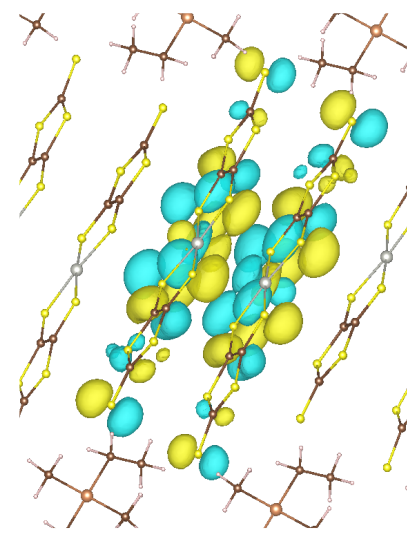

(f)

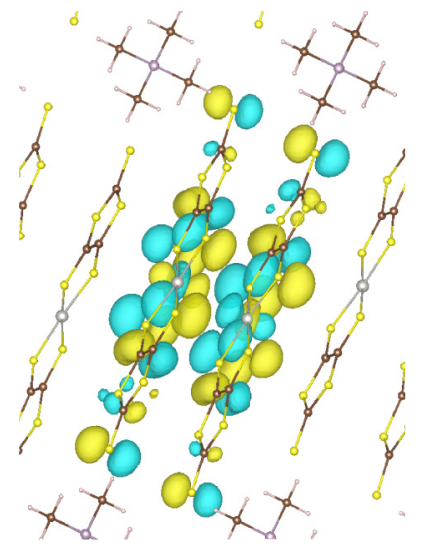

FIG. 1. Band dispersion of $\beta^{\prime}$-EtMe 3 Sb[Pd(dmit) $]_{2}$ at (a) $5 \mathrm{~K}$ and (b) RT. The solid lines are obtained by DFT calculations, while the broken lines are obtained using the maximally localized Wannier functions (MLWFs). The energy is shifted so that the Fermi energy is zero. (c) MLWF of $\beta^{\prime}-\mathrm{EtMe}_{3} \mathrm{Sb}\left[\mathrm{Pd}(\mathrm{dmit})_{2}\right]_{2}$ at $5 \mathrm{~K}$. Band dispersion of $\beta^{\prime}-\mathrm{Me}_{4} \mathrm{P}\left[\mathrm{Pd}(\mathrm{dmit})_{2}\right]_{2}$ at (d) $8 \mathrm{~K}$ and (e) RT, and (f) MLWF of $\beta^{\prime}-\mathrm{Me}_{4} \mathrm{P}\left[\mathrm{Pd}(\mathrm{dmit})_{2}\right]_{2}$ at $8 \mathrm{~K}$. MLWFs are drawn by using VESTA [42].

TABLE I. List of parameters obtained by the downfolding method in the dimer model (single-orbital extended Hubbard-type Hamiltonian) for $\beta^{\prime}-X\left[\mathrm{Pd}(\mathrm{dmit})_{2}\right]_{2}$. The values of the parameters at RT and those for EtMe 3 Sb at $4 \mathrm{~K}$ are the same as in Ref. [20]. The subscripts $a, b$, and $c$ represent the directions of the off-site transfer integrals and interactions, which are illustrated in Fig. 2(d).

\begin{tabular}{|c|c|c|c|c|c|c|c|c|c|c|c|}
\hline Cation & Temperature & $t_{a}(\mathrm{meV})$ & $t_{b}(\mathrm{meV})$ & $t_{c}(\mathrm{meV})$ & $U(\mathrm{eV})$ & $V_{a}(\mathrm{eV})$ & $V_{b}(\mathrm{eV})$ & $V_{c}(\mathrm{eV})$ & $J_{a}(\mathrm{meV})$ & $J_{b}(\mathrm{meV})$ & $J_{c}(\mathrm{meV})$ \\
\hline \multirow[t]{2}{*}{$\mathrm{Me}_{4} \mathrm{P}$} & RT & 59.3 & 44.0 & 30.9 & 0.883 & 0.449 & 0.465 & 0.413 & 3.26 & 2.54 & 1.29 \\
\hline & $8 \mathrm{~K}$ & 60.6 & 51.8 & 30.8 & 0.793 & 0.414 & 0.427 & 0.379 & 4.39 & 3.31 & 1.50 \\
\hline \multirow[t]{2}{*}{$\mathrm{Me}_{4} \mathrm{As}$} & RT & 54.9 & 41.5 & 33.8 & 0.864 & 0.426 & 0.442 & 0.394 & 2.77 & 2.37 & 1.35 \\
\hline & $5 \mathrm{~K}$ & 63.0 & 49.5 & 30.7 & 0.798 & 0.414 & 0.428 & 0.381 & 4.41 & 3.31 & 1.52 \\
\hline \multirow[t]{2}{*}{$\mathrm{Me}_{4} \mathrm{Sb}$} & RT & 49.7 & 36.5 & 38.9 & 0.898 & 0.429 & 0.444 & 0.402 & 2.67 & 1.75 & 1.49 \\
\hline & $5 \mathrm{~K}$ & 56.1 & 45.7 & 35.8 & 0.824 & 0.411 & 0.428 & 0.385 & 3.58 & 2.76 & 1.66 \\
\hline $\mathrm{EtMe}_{3} \mathrm{P}$ & RT & 56.1 & 42.0 & 35.0 & 0.889 & 0.442 & 0.460 & 0.411 & 2.88 & 2.02 & 1.48 \\
\hline $\mathrm{EtMe}_{3} \mathrm{As}$ & RT & 53.7 & 40.9 & 37.2 & 0.889 & 0.436 & 0.456 & 0.409 & 2.60 & 1.84 & 1.45 \\
\hline \multirow[t]{3}{*}{$\mathrm{EtMe}_{3} \mathrm{Sb}$} & $\mathrm{RT}$ & 48.8 & 35.6 & 41.7 & 0.906 & 0.427 & 0.449 & 0.406 & 3.21 & 2.23 & 1.40 \\
\hline & $5 \mathrm{~K}$ & 58.3 & 45.4 & 40.6 & 0.847 & 0.417 & 0.438 & 0.394 & 3.30 & 2.62 & 1.73 \\
\hline & $4 \mathrm{~K}$ & 57.1 & 44.6 & 40.3 & 0.840 & 0.413 & 0.434 & 0.390 & 2.23 & 1.64 & 1.71 \\
\hline $\mathrm{Et}_{2} \mathrm{Me}_{2} \mathrm{P}$ & $\mathrm{RT}$ & 53.4 & 38.5 & 38.6 & 0.947 & 0.478 & 0.497 & 0.450 & 2.51 & 2.32 & 1.43 \\
\hline \multirow[t]{2}{*}{$\mathrm{Et}_{2} \mathrm{Me}_{2} \mathrm{As}$} & RT & 50.2 & 36.3 & 38.7 & 0.923 & 0.422 & 0.467 & 0.448 & 3.30 & 2.63 & 1.73 \\
\hline & $5 \mathrm{~K}$ & 55.6 & 43.8 & 36.8 & 0.851 & 0.431 & 0.449 & 0.406 & 3.60 & 2.71 & 1.65 \\
\hline $\mathrm{Et}_{2} \mathrm{Me}_{2} \mathrm{Sb}$ & $\mathrm{RT}$ & 48.3 & 33.5 & 45.3 & 0.962 & 0.461 & 0.485 & 0.443 & 2.41 & 1.77 & 1.58 \\
\hline
\end{tabular}


listed in Table I. In this study, we evaluate all the microscopic parameters in the effective Hamiltonians such as the transfer integrals $t_{i j}$, on-site Coulomb interaction $U$, off-site Coulomb interaction $V_{i j}$, and direct exchange interactions $J_{i j}$ in an $a b$ initio way.

We note that the obtained Hamiltonians are three dimensional. Since the dmit salts are quasi-two-dimensional compounds, eliminating the weak three dimensionality is justified, and this greatly reduces the numerical cost of analyzing the Hamiltonians. In a previous study [43], the dimensional downfolding method was proposed to obtain two-dimensional Hamiltonians by eliminating the three dimensionality. It is shown that dimensional downfolding induces a constant shift $\Delta_{\text {DDF }}$ in the on-site and off-site Coulomb interactions $[29,43]$. Based on the previous study, in the analysis of the Hamiltonians, we take a constant shift $\Delta_{\mathrm{DDF}}=0.30 \mathrm{eV}$, which is a value comparable to that obtained for $\mathrm{EtMe}_{3} \mathrm{Sb}$ salt [29], for all compounds.

\section{B. Analysis of effective Hamiltonians}

To clarify how the differences in the low-energy effective Hamiltonians affect the physical quantities, we analyze the ground states of the low-energy effective Hamiltonians for nine dmit salts with RT structures and five dmit salts with LT structures. We take $t_{i j}, V_{i j}$, and $J_{i j}$ up to the next-nearest neighbor. To analyze the ground states of the target compounds, it is desirable to use the crystal structure at the lowest temperature. However, to obtain information on how structural differences affect electronic properties, it is useful to analyze the effective Hamiltonians for the RT structure even if they are hypothetical structures. For example, as we detail below, shrinking of the lattice parameters is essential for realizing quantum spin liquid behavior in $\mathrm{EtMe}_{3} \mathrm{Sb}$ salt.

To obtain the ground states, we use the locally optimal block conjugate gradient (LOBCG) method [44], which is implemented in $H \Phi$ [45]. In this study, we employ a $4 \times 4$ system size with twisted boundary conditions. In the strongly correlated region, the finite-size effects are expected to be small. However, lowering the temperature increases the system's weakly correlated region and the finite-size effects become significant. As we show later, we find that the amplitudes of the spin structure factors depend on the boundary conditions for $\mathrm{Me}_{4} \mathrm{P}$ salt in a LT structure, which has a relatively small $U / t_{a}$. To reduce the finite-size effects, we perform a boundary condition average [33] by introducing the flux $\phi$ as follows:

$$
\begin{gathered}
c_{i \sigma}^{\dagger} \rightarrow c_{i \sigma}^{\dagger} e^{i \boldsymbol{\phi} \cdot \boldsymbol{r}_{i}}, \\
c_{i \sigma} \rightarrow c_{i \sigma} e^{-i \boldsymbol{\phi} \cdot \boldsymbol{r}_{i}}, \\
\boldsymbol{\phi}=\left(\phi_{x}, \phi_{y}\right),
\end{gathered}
$$

where $\boldsymbol{r}_{i}$ is the position vector. From this flux insertion, the hopping terms and the pair-hopping terms change as follows:

$$
\begin{aligned}
c_{i \sigma}^{\dagger} c_{j \sigma} & \rightarrow c_{i \sigma}^{\dagger} c_{j \sigma} e^{i \boldsymbol{\phi} \cdot\left(\boldsymbol{r}_{i}-\boldsymbol{r}_{j}\right)}, \\
c_{i \sigma}^{\dagger} c_{j \rho} c_{i \rho}^{\dagger} c_{j \rho} & \rightarrow c_{i \sigma}^{\dagger} c_{j \rho} c_{i \rho}^{\dagger} c_{j \rho} e^{2 i \boldsymbol{\phi} \cdot\left(\boldsymbol{r}_{i}-\boldsymbol{r}_{j}\right)} .
\end{aligned}
$$

We note that the flux insertion does not change the Coulomb interactions.

In a previous study [33], it was shown that the finite-size effects are significantly reduced by averaging the physical quantities over $\phi$. In our calculations, we take $-\pi \leqslant \phi_{x}<$ $\pi$ and $0 \leqslant \phi_{y} \leqslant \pi$ with a division width of $\pi / 8$. In total, we calculate $N_{\phi}=16 \times 9=144$ boundary conditions. The boundary averaged spin structure factors are defined as

$$
\begin{gathered}
S(\boldsymbol{q}, \boldsymbol{\phi})=\frac{1}{N_{s}} \sum_{i, j}\left\langle\boldsymbol{S}_{i} \cdot \boldsymbol{S}_{j}\right\rangle_{\boldsymbol{\phi}} e^{i \boldsymbol{q}\left(\boldsymbol{r}_{i}-\boldsymbol{r}_{j}\right)}, \\
S_{\mathrm{ave}}(\boldsymbol{q})=\frac{1}{N_{\phi}} \sum_{\phi_{x}, \phi_{x}} S(\boldsymbol{q}, \boldsymbol{\phi}), \\
S\left(\boldsymbol{q}_{\text {peak }}\right)=\max _{\boldsymbol{q}}\left[S_{\mathrm{ave}}(\boldsymbol{q})\right],
\end{gathered}
$$

where $\langle\cdots\rangle_{\phi}$ means the expectation value of the ground state for the given boundary condition $\phi . N_{\mathrm{s}}$ represents the number of sites. The uncertainties in the boundary average are estimated as the standard errors $\sigma / \sqrt{N_{\phi}}$, where $\sigma$ is the standard deviation in the boundary average.

We also calculate the charge gap $\Delta_{\mathrm{c}}$ as follows:

$$
\begin{aligned}
& \mu^{+}(\boldsymbol{\phi})= \frac{E_{0}\left(N_{\mathrm{s}}+2, \boldsymbol{\phi}\right)-E_{0}\left(N_{\mathrm{s}}, \boldsymbol{\phi}\right)}{2}, \\
& \mu^{-}(\boldsymbol{\phi})= \frac{E_{0}\left(N_{\mathrm{s}}, \boldsymbol{\phi}\right)-E_{0}\left(N_{\mathrm{s}}-2, \boldsymbol{\phi}\right)}{2}, \\
& \mu_{\min }^{+}=\min _{\boldsymbol{\phi}} \mu^{+}(\boldsymbol{\phi}), \\
& \mu_{\max }^{-}=\max _{\boldsymbol{\phi}} \mu^{-}(\boldsymbol{\phi}), \\
& \Delta_{\mathrm{c}}=\max \left\{\mu_{\min }^{+}-\mu_{\max }^{-}, 0\right\},
\end{aligned}
$$

where $E_{0}(N, \boldsymbol{\phi})$ is the ground-state energy of a system with $N$ electrons and flux $\boldsymbol{\phi}$. The previous study showed that this definition of the charge gap can distinguish metallic and insulating phases well even for small system sizes [33]. It was also found that the absolute values of the charge gaps are slightly overestimated due to the finite-size effects.

\section{NUMERICAL RESULTS}

In this section, we first show the effective ab initio Hamiltonians for $\beta^{\prime}-\mathrm{X}\left[\mathrm{Pd}(\mathrm{dmit})_{2}\right]_{2}$ (X represents monovalent cations $\mathrm{Me}_{4} \mathrm{Y}, \mathrm{EtMe}_{3} \mathrm{Y}$, and $\mathrm{Et}_{2} \mathrm{Me}_{2} \mathrm{Y}$, where $\mathrm{Y}$ is a pnictogen, $\mathrm{P}$, $\mathrm{As}$, or $\mathrm{Sb}$ ). The values of the parameters such as the transfer integrals and Coulomb interactions for each compound are summarized in Table I. Next, we present the numerical results of solving effective Hamiltonians using the exact diagonalization method.

\section{A. Temperature dependence of effective Hamiltonians for $\beta^{\prime}-\mathrm{X}\left[\mathrm{Pd}(\mathrm{dmit})_{2}\right]_{2}$}

Figures 1(a) and 1(b) show band dispersions of $\mathrm{EtMe}_{3} \mathrm{Sb}$ salt for (a) $5 \mathrm{~K}$ and (b) RT structures, respectively. The solid lines are band structures directly calculated by DFT calculations, while the broken lines are those obtained from the transfer integrals between MLFWs defined in Eq. (1). Note that the two bands crossing the Fermi energy arise due to the presence of the two dimers in the primitive cell. Focusing on 
(a)

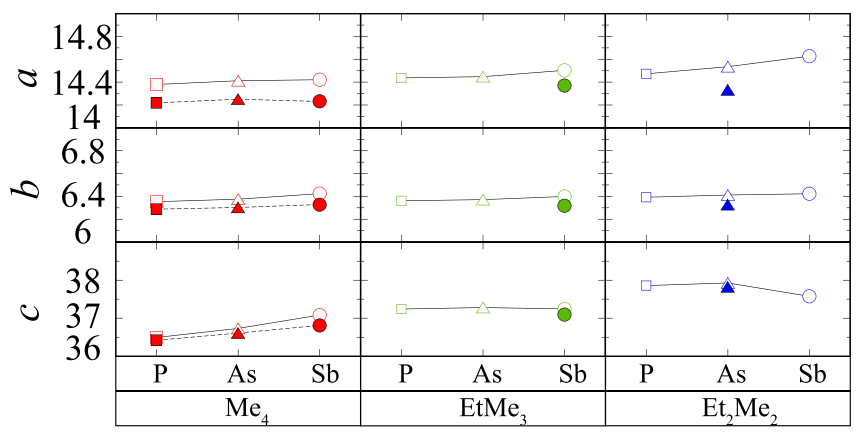

(c)

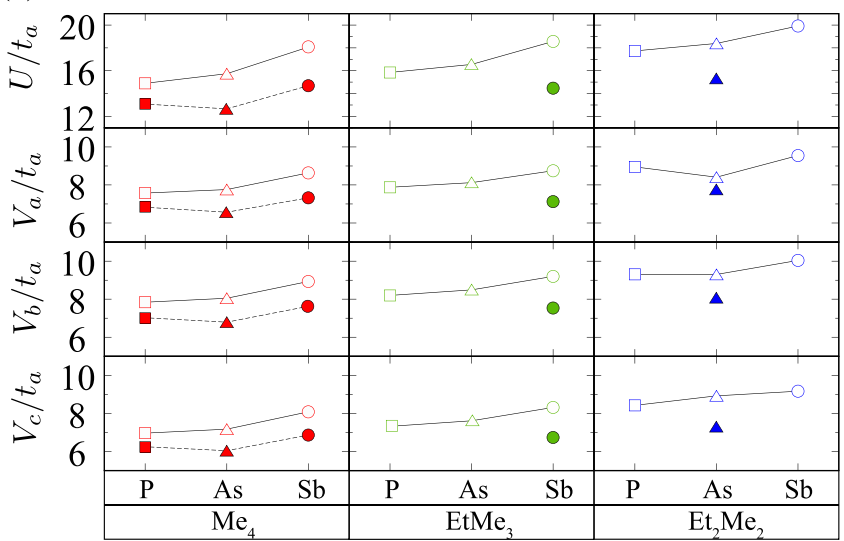

(b)

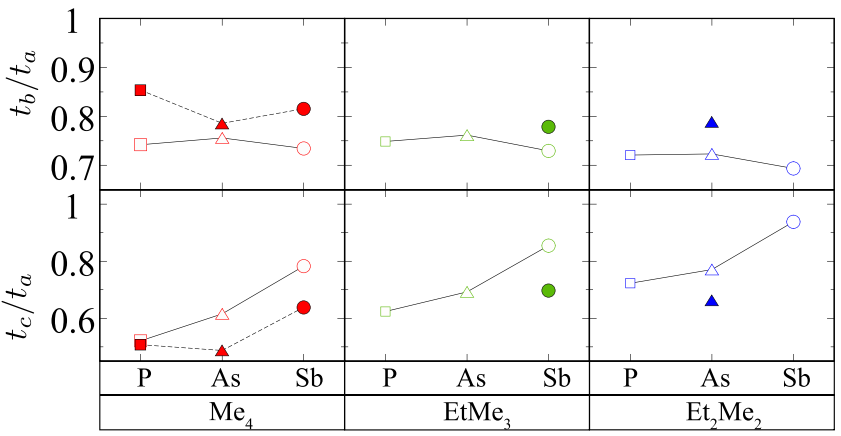

(d)

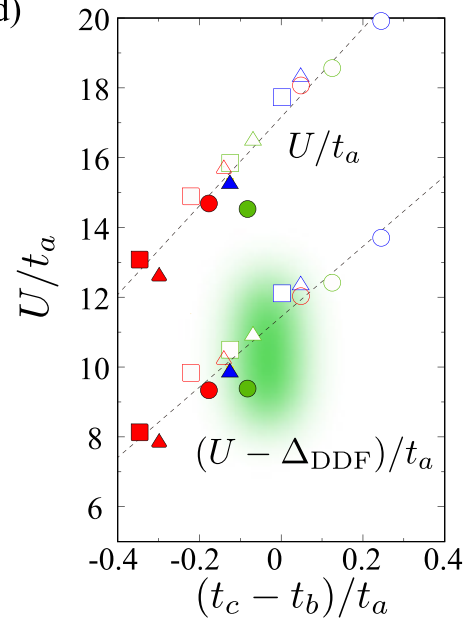

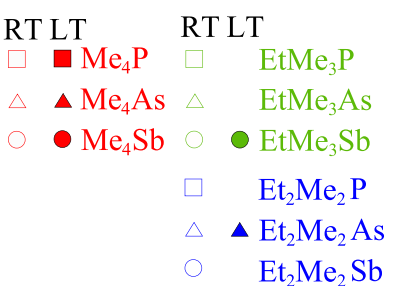

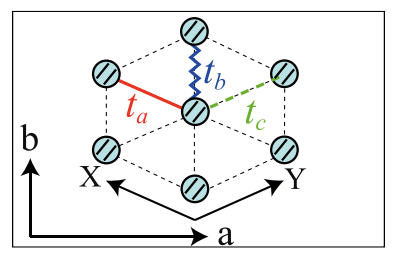

FIG. 2. (a) Lattice constants, (b) transfer integrals, and (c) Coulomb interactions for quasi-two-dimensional organic molecules $\beta^{\prime}$ $\mathrm{X}\left[\mathrm{Pd}(\mathrm{dmit})_{2}\right]_{2}$ (monovalent cations $\mathrm{X}=\mathrm{Me}_{4} \mathrm{Y}, \mathrm{EtMe}_{3} \mathrm{Y}$, and $\mathrm{Et}_{2} \mathrm{Me}_{2} \mathrm{Y}$, where the pnictogen $\mathrm{Y}$ is $\mathrm{P}, \mathrm{As}$, or $\mathrm{Sb}$ ). The open and closed symbols represent the data obtained using X-ray structures at RT and LT, respectively. (d) Compound dependence of $U / t_{a}$, $(U-\Delta \mathrm{DDF}) / t_{a}$, and $\left(t_{c}-t_{b}\right) / t_{a}$. The inset shows the schematic of the lattice structure. The closed circles indicate MLWFs and each bold line in the closed circles shows a dmit molecule.

the bands that cross the Fermi energy and the closest bands above and below them, we see that the band width increases, i.e., the band crossing the Fermi energy increases about $10 \%$ from RT to the lowest temperatures. This increase in the band width is due to the increase in the transfer integrals of $t_{a}$ and $t_{b}$ as listed in Table I, and caused by the lattice shrinking with decreasing temperature, as described later. In Fig. 1(c), the MLFW in $\mathrm{EtMe}_{3} \mathrm{Sb}$ salt at $5 \mathrm{~K}$ is depicted. It can be seen that the MLFW is formed as a dimer unit consisting of two $\left[\mathrm{Pd}(\mathrm{dmit})_{2}\right]$ monomers.

For comparison, the band structures of the LT and RT structures of the $\mathrm{Me}_{4} \mathrm{P}$ salt are shown in Figs. 1(d) and 1(e). We also find an approximately $8 \%$ enhancement of the band width of the low-energy band toward low temperatures. Unlike other $\beta^{\prime}$ salt, as the temperature decreases, only $t_{b}$ increases, while $t_{a}$ and $t_{c}$ hardly change in $\mathrm{Me}_{4} \mathrm{P}$ salt. Lastly, we also show that the MLFW in $\mathrm{Me}_{4} \mathrm{P}$ salt is formed as a dimer unit, similar to $\mathrm{EtMe}_{3} \mathrm{Sb}$ salt, as plotted in Fig. 1(f).

Next, the compound and temperature dependence of the transfer integrals are described. Figure 2(a) shows the lattice constants of each compound. At RT (open symbol), the lattice constants tend to increase in the order of $\mathrm{P}, \mathrm{As}$, and $\mathrm{Sb}$ for each cation family $\left(\mathrm{Me}_{4} \mathrm{Y}, \mathrm{EtMe}_{3} \mathrm{Y}\right.$, and $\left.\mathrm{Et}_{2} \mathrm{Me}_{2} \mathrm{Y}\right)$. As the temperature decreases, the lattice generally shrinks, so the overall lattice constant tends to decrease. In fact, the lattice constants get smaller. Figure 2(b) shows the transfer integrals of each compound. At RT (open symbols), $t_{c} / t_{a}$ tends to increase in the order $\mathrm{P}, \mathrm{As}$, and $\mathrm{Sb}$, while $t_{b} / t_{a}$ does not greatly depend on the pnictogen. It can also be seen that $t_{b}$ does not change much when the cation family is changed at the same temperature, while $t_{c}$ tends to increase in the order $\mathrm{Me}_{4} \mathrm{Y}, \mathrm{EtMe}_{3} \mathrm{Y}$, and $\mathrm{Et}_{2} \mathrm{Me}_{2} \mathrm{Y}$. As seen from Table I, $t_{a}$ and $t_{b}$ increase, while $t_{c}$ decreases at low temperatures compared to RT. Since the rate of change is larger for $t_{b}$ than $t_{c}, t_{b} / t_{a}$ increases while $t_{c} / t_{a}$ decreases. This indicates that the two dimensionality becomes stronger at low temperature. The cation and temperature dependencies of the transfer integrals coincide with the previous studies where the transfer integrals are obtained by the extended Hückel method [21,30]. It has been reported that the changes in $t_{a}$ and $t_{b}$ are mainly due to the distance between the dimers, while the change in $t_{c}$ is due to the degree of freedom of the atoms in the dimer.

In molecular solids, the Coulomb interactions tend to be relatively large because the molecules are bound by van der Waals interactions and the transfer integrals become small. It is worth noting here that the electron cloud spreads into the molecule. As a result, the on-site Coulomb interaction on the molecule is relaxed. On the other hand, long-range Coulomb interactions between molecules are less affected. Therefore, the long-range Coulomb interaction 
remains large in organic compounds and needs to be taken into account. From this point of view, we focus on the compounds and structure dependence of the screened Coulomb interactions. In Fig. 2(c), the effective Coulomb interactions $\left(U / t_{a}, V_{a} / t_{a}, V_{b} / t_{a}, V_{c} / t_{a}\right)$ are shown. At RT (open symbols), the effective Coulomb interactions gradually increase in the order $\mathrm{P}, \mathrm{As}$, and $\mathrm{Sb}$, as well as the transition integral $t_{c} / t_{a}$. The cation family dependence of the effective Coulomb interaction shows the same trend as $t_{c} / t_{a}$, i.e., the effective Coulomb interaction increases in the order $\mathrm{Me}_{4} \mathrm{Y}, \mathrm{EtMe}_{3} \mathrm{Y}$, and $\mathrm{Et}_{2} \mathrm{Me}_{2} \mathrm{Y}$. As seen from Table I, $U$ is almost the same for each cation family. Thus the difference of $U / t_{a}$ in the same cation family is mainly due to the difference in $t_{a}$. Although $V$ varies in each cation family, the ratio of change for $t_{a}$ is larger, so $V / t_{a}$ gives the same trend as $U / t_{a}$. At low temperatures, the band width is broadened due to the increase of $t_{a}$. As a result, the screened effect becomes large and the screened Coulomb interactions $U, V_{a}, V_{b}$, and $V_{c}$ are reduced, as shown in Table I. The trend becomes more pronounced for the effective interactions due to the increase of $t_{a}$. As seen from Fig. 2(c), the effective Coulomb interactions at low temperatures are weakened by about $20 \%$ compared to those at RT.

In a previous study [20], we showed that the effective on-site Coulomb interaction $U / t_{a}$ and the anisotropy of the transfer integral $t_{c} / t_{a}$ are key parameters for understanding the magnetic ground states in dmit salts. In Fig. 2(d), the compound dependencies of $U / t_{a}$ and $\left(t_{c}-t_{b}\right) / t_{a}$ are shown. Here, we adopt $\left(t_{c}-t_{b}\right) / t_{a}$, a parameter indicating the magnetic anisotropy, instead of $t_{c} / t_{a}$. As mentioned before, $U$ and $t_{c}$ change in almost the same way, while $t_{b}$ remains the same among the cation families. Therefore, $U / t_{a}$ tends to be proportional to $\left(t_{c}-t_{b}\right) / t_{a}$. Furthermore, as seen from Table I, $t_{a}$ is larger and $U$ and $t_{c}$ are smaller at low temperatures compared to at RT. Therefore, the trend that $U / t_{a}$ and $\left(t_{c}-t_{b}\right) / t_{a}$ decrease with decreasing temperature is obtained. We also plot the $\left(t_{c}-t_{b}\right) / t_{a}$ dependence of $\left(U-\Delta_{\mathrm{DDF}}\right) / t_{a}$, where $\Delta_{\mathrm{DDF}}=0.30$ is a constant shift parameter to take into account considering the dimensional downfolding effect. It can be seen that the slope becomes a little more gradual, although the trend is basically the same as for $U / t_{a}$. As will be detailed in the next section, the magnetic order moment decreases in the effective Hamiltonians of materials toward $\left(t_{c}-t_{b}\right) / t_{a} \sim 0$ (shaded area), and a QSL-like behavior is seen.

Finally, we compare the transfer integrals and Coulomb interactions obtained in our calculations with those calculated in previous studies. For RT structures, our obtained values of transfer integrals derived from MLWFs reasonably agree with those calculated with a different DFT and fitting methods [23-25]. Similar to the cation dependence found in the RT structures, in the LT structure, $t_{b}$ is larger than $t_{c}$ for the salts that show AF order. This difference decreases in the compounds with lower Néel temperatures and, then, the two transfer energies become comparable around the $\mathrm{EtMe}_{3} \mathrm{Sb}$ salt, experimentally showing the QSL state [46]. For the magnitude of the Coulomb interactions, the cRPA calculation was performed for the $4 \mathrm{~K}$ structure of $\mathrm{EtMe}_{3} \mathrm{Sb}$ salt by Nakamura et al. [29] and the results before dimensional downfolding are in good agreement. In addition, $U / W$ is given by 1.94 in our calculation for $\mathrm{EtMe}_{3} \mathrm{Sb}$ salt at $5 \mathrm{~K}$, while, experimentally, it (a) $\mathrm{Me}_{4} \mathrm{P}$ (LT)

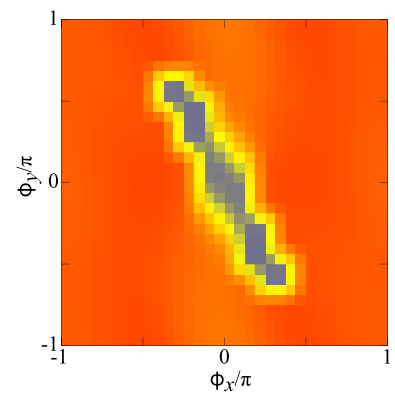

(b) $\mathrm{EtMe}_{3} \mathrm{Sb}$ (LT)

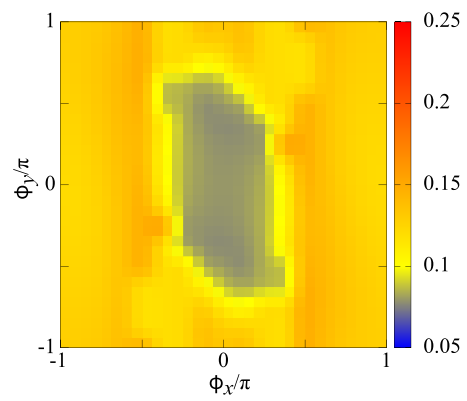

FIG. 3. Boundary-condition dependence of the peak value of the spin structure factors $\left[S\left(\boldsymbol{q}_{\text {peak }}, \boldsymbol{\phi}\right) / N_{\mathrm{s}}\right]$ for the effective Hamiltonians of (a) $\mathrm{Me}_{4} \mathrm{P}$ with an LT structure and (b) $\mathrm{EtMe}_{3} \mathrm{Sb}$ with an LT structure. For $\mathrm{Me}_{4} \mathrm{P}, \boldsymbol{q}_{\text {peak }}=(\pi, 0)$ for all the boundary conditions. Although $\boldsymbol{q}_{\text {peak }}=(\pi, 0)$ for EtMe 3 Sb for most boundary conditions, $\boldsymbol{q}_{\text {peak }}=(\pi, \pi / 2)$ in 30 boundary conditions. As we explained in Sec. II B, we calculate $-\pi \leqslant \phi_{x}<\pi$ and $0 \leqslant \phi_{y} \leqslant \pi$ with division width $\pi / 8$. Using the relations $S\left(\boldsymbol{q}_{\text {peak }}, \boldsymbol{\phi}\right)=S\left(\boldsymbol{q}_{\text {peak }},-\boldsymbol{\phi}\right)$ and $S\left(\boldsymbol{q}_{\text {peak }},\left(\phi_{x}=\pi, \phi_{y}\right)\right)=S\left(\boldsymbol{q}_{\text {peak }},\left(\phi_{x}=-\pi, \phi_{y}\right)\right)$, we plot the peak values in the whole Brillouin zone.

has been reported that $U / W$ is estimated as 2.3 [47], which is consistent with our results.

\section{B. Analysis of effective Hamiltonians}

Here, we solve the low-energy effective Hamiltonians for dmit salts using the exact diagonalization for the $4 \times 4$ cluster. As explained in Sec. II B, we employ a boundary-condition average to reduce the finite-size effects. As a typical example of boundary-condition dependence of the spin structure factors, we show $S\left(\boldsymbol{q}_{\text {peak }}, \boldsymbol{\phi}\right) / N_{\mathrm{s}}$ of the effective Hamiltonians for $\mathrm{Me}_{4} \mathrm{P}$ and EtMe 3 Sb salts with LT structures in Fig. 3. For both compounds, we find that the spin structure factors become small around $\boldsymbol{\phi}=\mathbf{0}$, i.e., periodic-periodic boundary conditions. For $\mathrm{Me}_{4} \mathrm{P}$ salt, except for a narrow region around $\boldsymbol{\phi}=\mathbf{0}$, the spin structure factors show a clear signature of stripe magnetic ordering $\left[\boldsymbol{q}_{\text {peak }}=(\pi, 0)\right]$. In contrast to this, $S\left(\boldsymbol{q}_{\text {pear }}, \boldsymbol{\phi}\right) / N_{\mathrm{s}}$ in $\mathrm{EtMe}_{3} \mathrm{Sb}$ salt is still small sufficiently far from $\boldsymbol{\phi}=\mathbf{0}$. This significant suppression of the spin structure factors for all the boundary conditions indicates the appearance of the quantum spin liquid state in $\mathrm{EtMe}_{3} \mathrm{Sb}$ salt.

In Fig. 4, we show $\mu^{+}(\boldsymbol{\phi})$ and $\mu^{-}(\boldsymbol{\phi})$ as functions of the flux $\phi$ for the effective Hamiltonians for $\mathrm{Me}_{4} \mathrm{P}$ and $\mathrm{EtMe}_{3} \mathrm{Sb}$ salts with LT structures. As explained in Sec. II B, the minimum value of the indirect gap between $\mu^{+}(\boldsymbol{\phi})$ and $\mu^{-}(\boldsymbol{\phi})$ is the charge gap $\Delta_{c}$. Since $\mu^{+}(\boldsymbol{\phi})$ and $\mu^{-}(\boldsymbol{\phi})$ do not overlap, the charge gap is finite and thus both compounds are in insulating phases. The estimated charge gap is $\Delta_{\mathrm{c}}=2.6 t_{a}$ for $\mathrm{Me}_{4} \mathrm{P}$ salt and $\Delta_{\mathrm{c}}=3.3 t_{a}$ for $\mathrm{EtMe}_{3} \mathrm{Sb}$ salt. This difference is mainly attributed to the amplitude of the Coulomb interactions. Since the Coulomb interactions of $\mathrm{Me}_{4} \mathrm{P}$ salt $\left[\left(U-\Delta_{\mathrm{DDF}}\right) / t_{a} \sim 8.2\right]$ are smaller than that of $\mathrm{EtMe}_{3} \mathrm{Sb}$ salt $\left[\left(U-\Delta_{\mathrm{DDF}}\right) / t_{a} \sim 9.5\right]$, the charge gap becomes small. In spite of the large Coulomb interactions, in $\mathrm{EtMe}_{3} \mathrm{Sb}$ salt, the geometrical frustration as well as the off-site Coulomb interaction melt the long-range magnetic order and induce the quantum spin liquid behavior. 
(a) $\mathrm{Me}_{4} \mathrm{P}$ (LT)
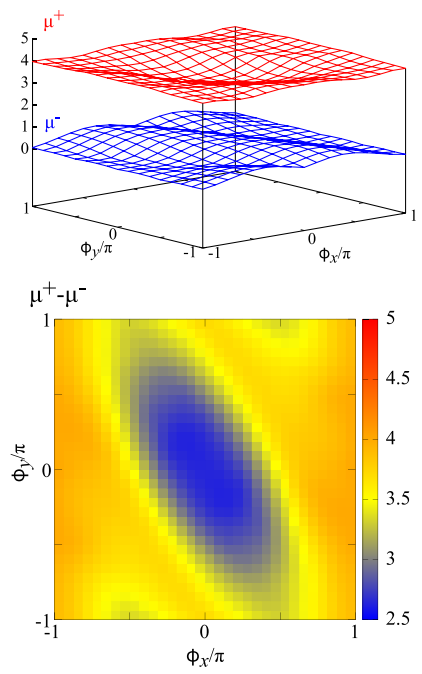

(b) $\mathrm{EtMe}_{3} \mathrm{Sb}$ (LT)
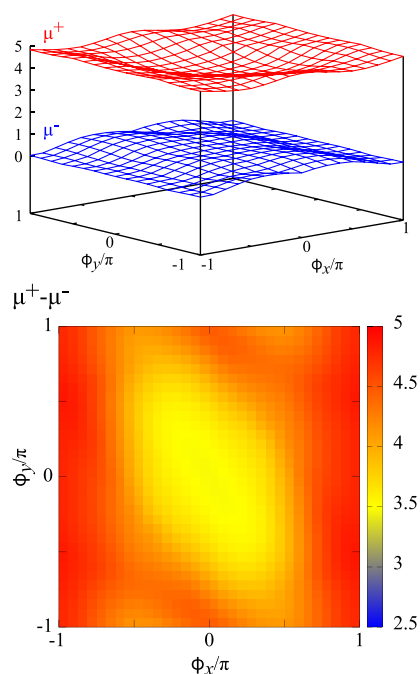

FIG. 4. Boundary-condition dependence of the chemical potentials $\mu^{+}(\boldsymbol{\phi})$ and $\mu^{-}(\boldsymbol{\phi})$ for the effective Hamiltonians of (a) $\mathrm{Me}_{4} \mathrm{P}$ salt with an LT structure and (b) $\mathrm{EtMe}_{3} \mathrm{Sb}$ salt with an LT structure. For clarity, the energy is shifted so that $\min _{\phi} \mu^{-}(\phi)$ is zero. In the bottom panel, $\mu^{+}(\boldsymbol{\phi})-\mu^{-}(\boldsymbol{\phi})$ is shown.

In the bottom panel of Fig. 4, we show $\mu^{+}(\boldsymbol{\phi})-\mu^{-}(\boldsymbol{\phi})$, which corresponds to the direct gap, i.e., the charge gap for each boundary condition. For both compounds, we find that the region where the direct gap becomes small is roughly consistent with the region where the magnetic ordered moment becomes small. Since the ground state of $\mathrm{Me}_{4} \mathrm{P}$ salt is a magnetically ordered phase, this correspondence is natural, i.e., a small magnetic ordered moment means a small charge gap. However, this correspondence still holds for $\mathrm{EtMe}_{3} \mathrm{Sb}$ salt, where the magnetic order is suppressed. The correspondence indicates that the gap generation mechanism in the quantum spin liquid is still closely related with the development of the spin correlations.

Here, we discuss the compound dependence of the boundary-averaged physical quantities. First, we calculated the charge structure factors and confirmed that a characteristic peak does not appear and thus all compounds are not in the charge ordered state. Thus, in the following, we only focus on the spin state. In Fig. 5(a), we show the peak values of the boundary averaged spin structure factors $\left[S\left(\boldsymbol{q}_{\text {peak }}\right)\right]$. All the spin structure factors in momentum space are shown in Fig. 6. Consistent with our previous study, in $\mathrm{EtMe}_{3} \mathrm{Sb}$ salt, the spin structure factors are significantly reduced even when we perform the boundary average. This reduction is consistent with the quantum spin liquid behavior observed in experiments. Moreover, by performing the boundary average, we find that the spin structure factors in $\mathrm{EtMe}_{3} \mathrm{Sb}$ salt have weak $q_{y}$ dependence as shown in Fig. 6, i.e., implying a larger anisotropy between $x$ and $y$ directions than other compounds, although the parameters of the transfer integrals and the interactions have no apparent differences in anisotropy compared to those of other compounds. It is an intriguing future subject to clarify whether this trend survives in larger sized systems.
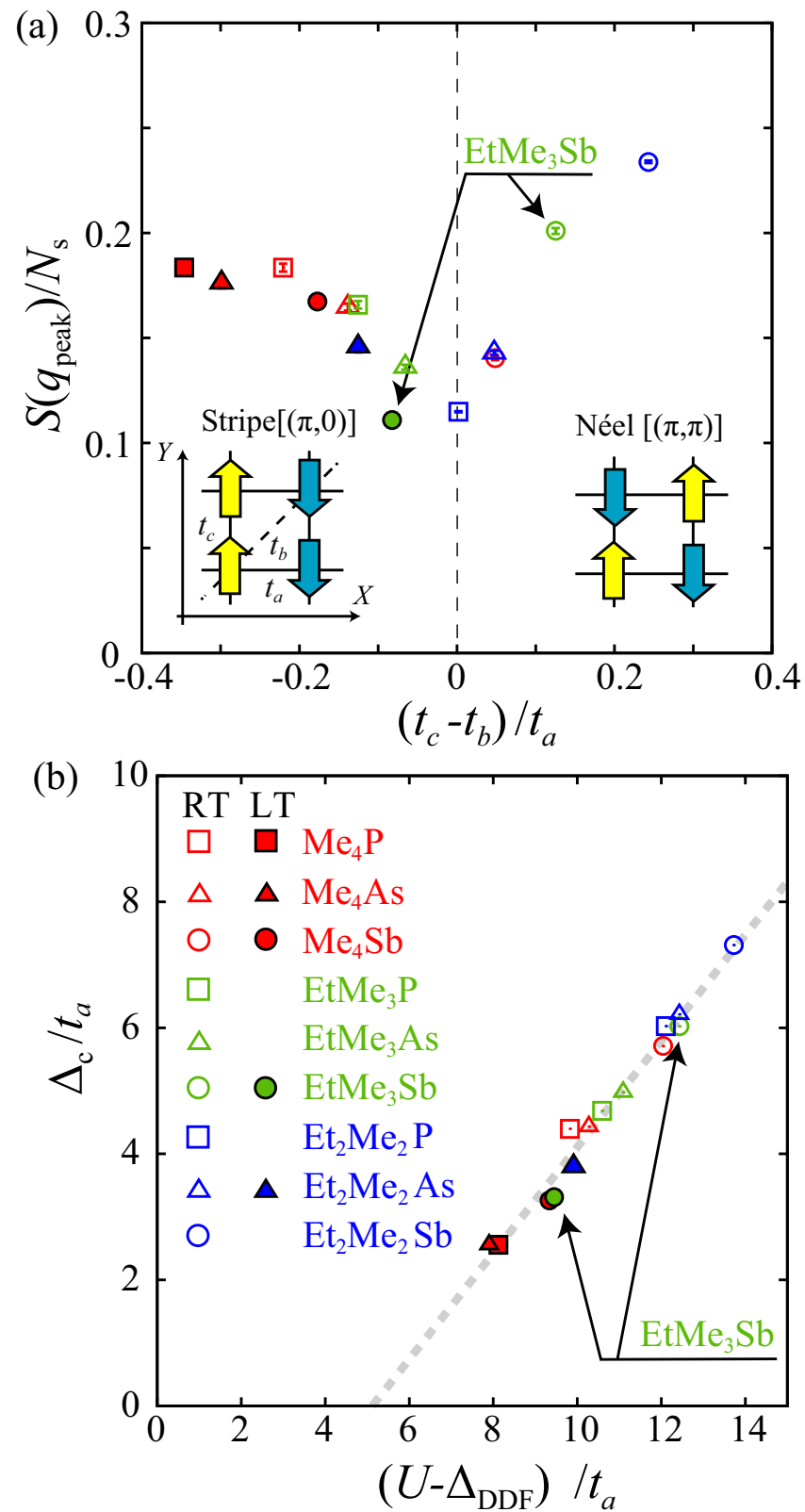

FIG. 5. (a) Compound dependence of the peak values of the boundary averaged spin structure factors $S\left(\boldsymbol{q}_{\text {peak }}\right) / N_{\mathrm{s}}$ as a function of $\left(t_{c}-t_{b}\right) / t_{a}$. For $\left(t_{c}-t_{b}\right) / t_{a} \ll 0$, the stripe magnetic ordered phase becomes stable, while the Néel-type magnetic ordered phase becomes stable for $\left(t_{c}-t_{b}\right) / t_{a} \gg 0$. Sandwiched by two magnetic ordered phases, around $\left(t_{c}-t_{b}\right) / t_{a}=0$, the spin structure factors are significantly reduced. The error bars are estimated as the standard errors of the boundary average (see main text). (b) Compound dependence of the charge gap as a function of $\left(U-\Delta_{\mathrm{DDF}}\right) / t_{a}$. The on-site-Coulomb dependence of the charge gap is fitted by the function $\alpha\left(U / t_{a}-U_{c} / t_{a}\right)$, where $\alpha=0.84(3)$ and $U_{c} / t_{a}=5.1(2)$. The broken line is the result of the fitting.

We examine how the shrinkage of the lattice constants associated with lowering temperature affects the spin correlations. As shown in Fig. 6, for all the LT structures, we find that stripe-type spin correlations $\left[q_{\text {peak }}=(\pi, 0)\right]$ become dominant since all the compounds are located at $\left(t_{c}-t_{b}\right) / t_{a}<$ 


\section{Room temperature}
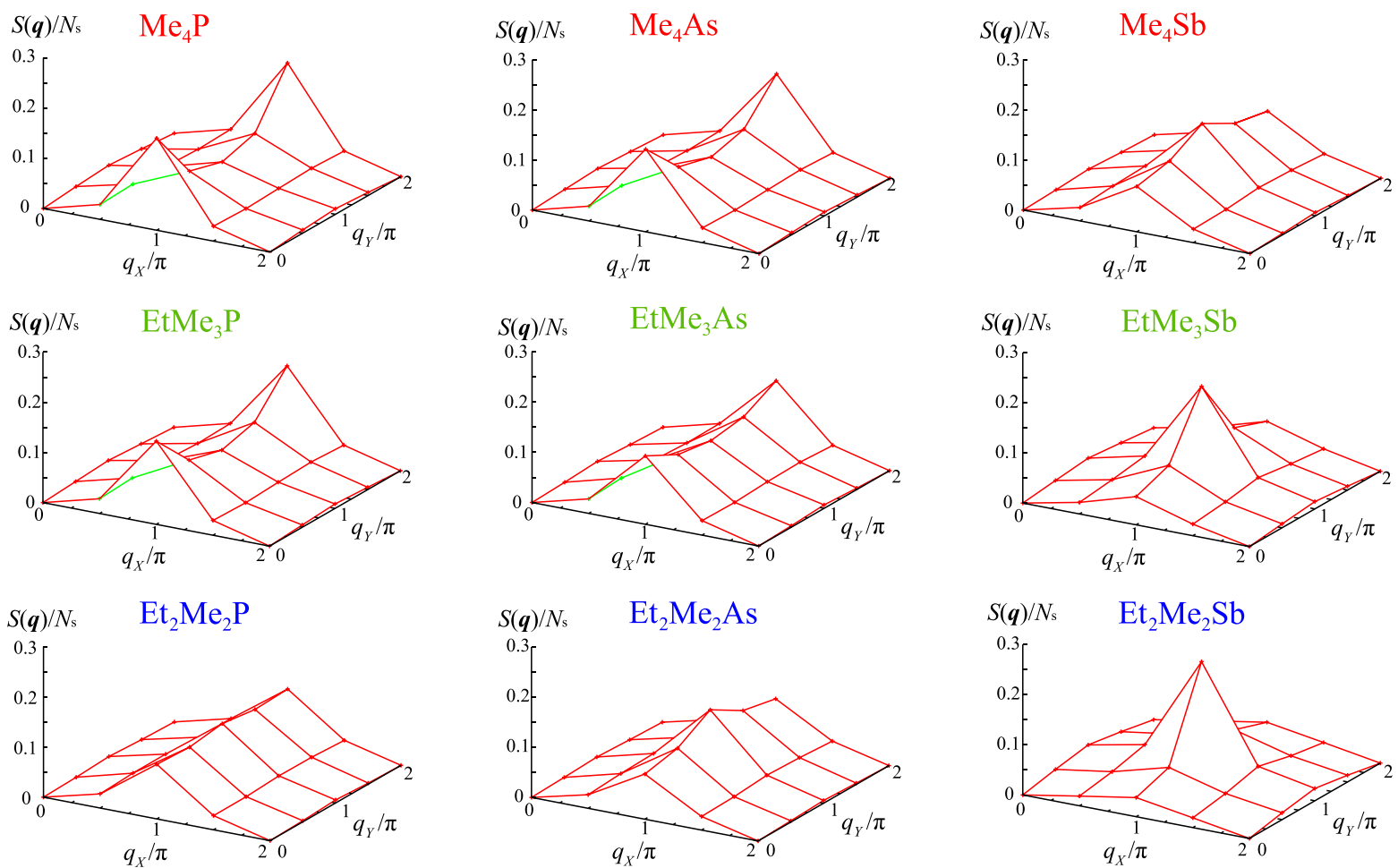

\section{Low temperature}
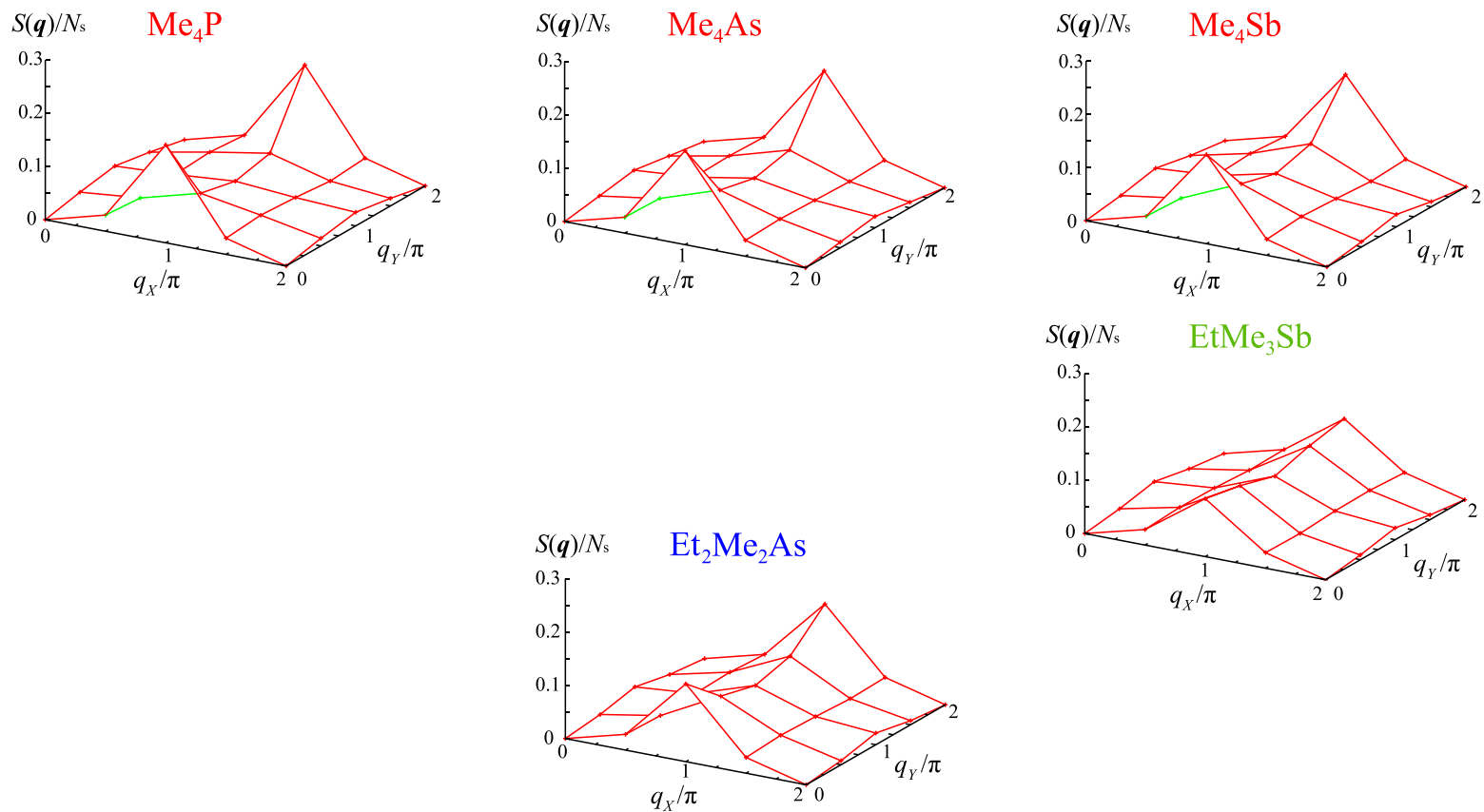

FIG. 6. Boundary-averaged spin structure factors for RT structures (upper panel) and LT structures (lower panel).

0 , while Néel-type spin correlations $\left[q_{\text {peak }}=(\pi, \pi)\right]$ become dominant in several RT structures. For example, in $\mathrm{Me}_{4} \mathrm{Sb}$ salt, although the Néel-type spin correlation $\left[\boldsymbol{q}_{\text {peak }}=(\pi, \pi)\right]$ is dominant in the effective Hamiltonians for the RT structure, the stripe spin correlation $\left[\boldsymbol{q}_{\text {peak }}=(\pi, 0)\right]$ becomes dominant in the effective Hamiltonians for the LT structure. This result indicates that we can observe changes in the spin correlations by lowering the temperature in $\mathrm{Me}_{4} \mathrm{Sb}$ by measuring the directions of the spin correlations.

We next examine the compound dependence of the charge gap. As shown in Fig. 5(b), for the effective Hamiltonians for LT structures, we find that the charge gap is significantly 
reduced because decreasing temperature decreases $U / t_{a}$. For example, in $\mathrm{EtMe}_{3} \mathrm{Sb}$ salt, $\Delta_{c} \sim 6 t_{a} \sim 0.29 \mathrm{eV}$ changes to $\Delta_{c} \sim 3.3 t_{a} \sim 0.18 \mathrm{eV}$. This result indicates that $\mathrm{EtMe}_{3} \mathrm{Sb}$ salt approaches the boundary of the metal-insulator transition as the temperature is lowered. We note that the charge gap at LT $\left(\Delta_{c} \sim 0.18 \mathrm{eV}\right)$ is roughly consistent with the experimental value $\Delta_{c}=650 \mathrm{~cm}^{-1} \sim 0.08 \mathrm{eV}$ estimated by the optical conductivity [47]. The discrepancy $(\sim 0.1 \mathrm{eV})$ can be attributed to the finite-size effects since the charge gap is expected to be smaller as the system size increases.

\section{SUMMARY AND DISCUSSION}

In this paper, we have derived low-energy effective Hamiltonians for nine dmit salts with RT structures and five dmit salts with LT structures. We have found that both $U / t_{a}$ and $\left(t_{c}-t_{b}\right) / t_{a}$ decrease with decreasing temperature. We have obtained the ground states of the effective Hamiltonians using the exact diagonalization method with a boundary condition average, which reduces the finite size effects. The results show that the charge gap remains finite in all the materials, although it decreases with decreasing temperature due to the decrease in $U / t_{a}$. By calculating the spin structure factors, we have found that stripe-type spin correlations appear in all the effective Hamiltonians for LT structures, while some of the effective Hamiltonians for the RT structure show Néel-type spin correlations. The peak values of the spin structure factors are reduced around $\mathrm{EtMe}_{3} \mathrm{Sb}$ salt for LT structures, because $\left(t_{c}-t_{b}\right) / t_{a} \sim 0$ is satisfied in the effective model of $\mathrm{EtMe}_{3} \mathrm{Sb}$ salt. This result indicates that geometrical frustration in the interchain couplings is a key to inducing the quantum spin liquid behavior observed in $\mathrm{EtMe}_{3} \mathrm{Sb}$ salt. We note that it has been proposed that interchain magnetic frustration plays an important role inducing QSL in the frustrated Hubbard model [26] and the Heisenberg model $[46,48,49]$. Further theoretical calculations to clarify the differences and common features between the QSL in the ab initio Hamiltonians and that in the simplified Hubbard/Heisenberg model remain as an intriguing subject of study but are beyond the scope of this paper. This issue will be studied and presented elsewhere [50].

Here, we comment on the validity of the dimer model description (i.e., single-orbital description) of the dmit salts. A recent nuclear magnetic resonance (NMR) experiment [51] showed that spin polarizations occur within dimers in $\mathrm{Me}_{4} \mathrm{P}$ and $\mathrm{Me}_{4} \mathrm{Sb}$ salts, where the antiferromagnetic ordered phase is the ground state. This result indicates that it is necessary to explicitly consider the interdimer degrees of freedom to explain the microscopic structures of antiferromagnetic ordered states. However, as we have shown in this study, the quantum spin liquid behavior is induced by frustration in the magnetic interactions among dimers. Regarding the reduction of the magnetic ordered moment, it is plausible that the microscopic structure of the spin moment within the dimers does not play an essential role. The success in reproducing the reduction of the magnetic ordered moment in $\mathrm{EtMe}_{3} \mathrm{Sb}$ salt demonstrates the validity of the dimer model descriptions of dmit salts at least for capturing the essence of the quantum spin liquid behaviors. A direct comparison and analysis of the dimer model and multiorbital models that explicitly include the interdimer degrees of freedom is an intriguing subject left for future studies.

The present analysis shows that the microscopic parameters in the effective Hamiltonians of the dmit salts are sensitive to lattice distortions. In fact, the values of the on-site Coulomb interaction and the charge gap for the LT structure are found to be reduced by about $20 \%$ compared to those for the RT structures. The amplitude of the geometrical frustration characterized by $\left(t_{c}-t_{b}\right) / t_{a}$ is also greatly affected by the changes in the lattice constants. By utilizing this flexibility, it may be possible to tune the ground states of dmit salts other than $\mathrm{EtMe}_{3} \mathrm{Sb}$ to become quantum spin liquids by controlling the lattice constants via hydrostatic/uniaxial pressure. Using $a b$ initio calculations, it is possible to analyze the pressure effects and predict a way to realize a quantum spin liquid. It is noted that the Néel ordered state has not been observed in $\mathrm{Et}_{2} \mathrm{Me}_{2} \mathrm{Sb}$ salt, although it has the largest positive $\left(t_{c}-t_{b}\right) / t_{a}$ at room temperature. This is because this salt undergoes the structure phase transition with the charge ordering. However, we do not find the clear signature of the experimentally observed charge ordered $(\mathrm{CO})$ in the effective Hamiltonians. This result indicates that the more complex effects such as more distant long-range Coulomb interactions and electron-phonon couplings should be taken into account [25] for reproducing the experimentally observed CO state. Nevertheless, if we can use the pressure-induced changes in the structures, it might be possible to realize the Néel ordered state at low temperatures instead of the $\mathrm{CO}$ state. If it is possible, we may be able to study the crossover of spin states, i.e., crossover from the Néel state to the stripe state. Detailed studies of the pressure effects for stabilizing the quantum spin liquid and possible superconducting phases under pressure are left as important challenges to be addressed.

\section{ACKNOWLEDGMENTS}

We wish to thank K. Ido, M. Imada, S. Fujiyama, and R. Kato for their helpful contributions. A part of the calculations was done using the Supercomputer Center, the Institute for Solid State Physics, the University of Tokyo. K.Y. and T.M. were supported by Building of Consortia for the Development of Human Resources in Science and Technology, MEXT, Japan. This work was also supported by a Grant-in-Aid for Scientific Research No. 21H01041 from the Ministry of Education, Culture, Sports, Science and Technology, Japan. This work was supported by MEXT as "Program for Promoting Researches on the Supercomputer Fugaku" (Basic Science for Emergence and Functionality in Quantum Matter-Innovative Strongly-Correlated Electron Science by Integration of "Fugaku" and Frontier Experiments-, JPMXP1020200104, Project ID No. hp210163).
[1] M. Imada, A. Fujimori, and Y. Tokura, Metal-insulator transitions, Rev. Mod. Phys. 70, 1039 (1998).
[2] L. Balents, Spin liquids in frustrated magnets, Nature (London) 464, 199 (2010). 
[3] T. Ishiguro and K. Yamaji, Organic Superconductors (Springer, New York, 1998).

[4] K. Kanoda, Metal-insulator transition in $\kappa$-(ET) $)_{2} X$ and $(\mathrm{DCNQI})_{2} M$ : Two contrasting manifestation of electron correlation, J. Phys. Soc. Jpn. 75, 051007 (2006).

[5] T. Koretsune and C. Hotta, Evaluating model parameters of the $\kappa$ - and $\beta^{\prime}$-type Mott insulating organic solids, Phys. Rev. B 89, 045102 (2014).

[6] K. Kanoda and R. Kato, Mott physics in organic conductors with triangular lattices, Annu. Rev. Condens. Matter Phys. 2, 167 (2011).

[7] S. Uji, M. Chaparala, S. Hill, P. S. Sandhu, J. Quails, L. Seger, and J. S. Brooks, Effective mass and combination frequencies of de Haas-van Alphen oscillations in $\kappa$-(BEDT-TTF $)_{2} \mathrm{Cu}(\mathrm{NCS})_{2}$, Synth. Met. 85, 1573 (1997).

[8] M. S. Hybertsen, E. B. Stechel, M. Schluter, and D. R. Jennison, Renormalization from density-functional theory to strong-coupling models for electronic states in $\mathrm{Cu}-\mathrm{O}$ materials, Phys. Rev. B 41, 11068 (1990).

[9] M. Hirayama, Y. Yamaji, T. Misawa, and M. Imada, Ab initio effective Hamiltonians for cuprate superconductors, Phys. Rev. B 98, 134501 (2018).

[10] E. Canadell, I. E.-I. Rachidi, S. Ravy, J. P. Pouget, L. Brossard, and J. P. Legros, On the band electronic structure of $\mathrm{X}\left[\mathrm{M}(\mathrm{dmit})_{2}\right]_{2}\left(\mathrm{X}=\mathrm{TTF},\left(\mathrm{CH}_{3}\right)_{4} \mathrm{~N} ; \mathrm{M}=\mathrm{Ni}, \mathrm{Pd}\right)$ molecular conductors and superconductors, J. Phys. (France) 50, 2967 (1989).

[11] E. Canadell, S. Ravy, J. P. Pouget, and L. Brossard, Concerning the band structure of $\mathrm{D}\left(\mathrm{M}(\mathrm{dmit})_{2}\right)_{2}\left(\mathrm{D}=\mathrm{TTF}, \mathrm{Cs}, \mathrm{NMe}_{4}\right) ; \mathrm{M}=$ $\mathrm{Ni}, \mathrm{Pd})$ molecular conductors and superconductors: Role of the $\mathrm{M}(\mathrm{dmit})_{2}$ HOMO and LUMO, Solid State Commun. 75, 633 (1990).

[12] R. Kato, Conducting metal dithiolene complexes: Structural and electronic properties, Chem. Rev. 104, 5319 (2004).

[13] T. Miyazaki and T. Ohno, First-principles study of the electronic structure of the organic solids $\left(\mathrm{CH}_{3}\right)_{4} \mathrm{~N}\left[\mathrm{M}(\mathrm{dmit})_{2}\right]_{2}(\mathrm{M}=\mathrm{Ni}$ and $\mathrm{Pd}$ ): Role of dimerization and the stability of the formation of a dimer, Phys. Rev. B 59, R5269 (1999).

[14] M. Tamura and R. Kato, Magnetic susceptibility of $\beta^{\prime}$ $\left[\mathrm{Pd}(\mathrm{dmit})_{2}\right]$ salts $(\mathrm{dmit}=1$, 3-dithiol-2-thione-4, 5-dithiolate, $\mathrm{C}_{3} \mathrm{~S}_{5}$ ): Evidence for frustration in spin-1/2 Heisenberg antiferromagnets on a triangular lattice, J. Phys.: Condens. Matter 14, L729 (2002).

[15] T. Itou, A. Oyamada, S. Maegawa, M. Tamura, and R. Kato, Quantum spin liquid in the spin-1/2 triangular antiferromagnet $\mathrm{EtMe}_{3} \mathrm{Sb}\left[\mathrm{Pd}(\mathrm{dmit})_{2}\right]_{2}$, Phys. Rev. B 77, 104413 (2008).

[16] S. Yamashita and Y. Nakazawa, Heat capacities of antiferromagnetic dimer-Mott insulators in organic charge-transfer complexes, J. Therm. Anal. Calorim. 99, 153 (2010).

[17] S. Yamashita, T. Yamamoto, Y. Nakazawa, M. Tamura, and R. Kato, Gapless spin liquid of an organic triangular compound evidenced by thermodynamic measurements, Nat. Commun. 2 , 275 (2011)

[18] A. Nakao and R. Kato, Structural study of low temperature charge-separated phases of $\mathrm{Pd}(\mathrm{dmit})_{2}$-based molecular conductors, J. Phys. Soc. Jpn. 74, 2754 (2005).

[19] M. Tamura and R. Kato, Valence instability in a dimer of twoorbital system: possible charge separation due to 'negative u' effect, Chem. Phys. Lett. 387, 448 (2004).
[20] T. Misawa, K. Yoshimi, and T. Tsumuraya, Electronic correlation and geometrical frustration in molecular solids: A systematic $a b$ initio study of $\beta^{\prime}-X\left[\operatorname{Pd}(\mathrm{dmit})_{2}\right]_{2}$, Phys. Rev. Res. 2, 032072(R) (2020).

[21] R. Kato and H. Cui, Cation dependence of crystal structure and band parameters in a series of molecular conductors, $\beta^{\prime}$-(cation) $\left[\mathrm{Pd}(\mathrm{dmit})_{2}\right]_{2}(\mathrm{dmit}=1,3$-dithiole-2-thione-4,5dithiolate), Crystals 2, 861 (2012).

[22] E. P. Scriven and B. J. Powell, Geometrical Frustration in the Spin Liquid $\beta^{\prime}-\mathrm{Me}_{3} \mathrm{EtSb}\left[\mathrm{Pd}(\mathrm{dmit})_{2}\right]_{2}$ and the ValenceBond Solid $\mathrm{Me}_{3} \mathrm{EtP}\left[\mathrm{Pd}(\mathrm{dmit})_{2}\right]_{2}$, Phys. Rev. Lett. 109, 097206 (2012).

[23] A. C. Jacko, L. F. Tocchio, H. O. Jeschke, and R. Valentí, Importance of anisotropy in the spin-liquid candidate $\mathrm{Me}_{3} \mathrm{EtSb}\left[\mathrm{Pd}(\mathrm{dmit})_{2}\right]_{2}$, Phys. Rev. B 88, 155139 (2013).

[24] T. Tsumuraya, H. Seo, M. Tsuchiizu, R. Kato, and T. Miyazaki, Cation dependence of the electronic states in molecular triangular lattice system $\beta^{\prime}-X\left[\operatorname{Pd}(\mathrm{dmit})_{2}\right]_{2}$ : A first-principles study, J. Phys. Soc. Jpn. 82, 033709 (2013).

[25] H. Seo, T. Tsumuraya, M. Tsuchiizu, T. Miyazaki, and R. Kato, Fragment model study of molecular multiorbital system $\mathrm{X}\left[\mathrm{Pd}(\mathrm{dmit})_{2}\right]_{2}$, J. Phys. Soc. Jpn. 84, 044716 (2015).

[26] L. F. Tocchio, C. Gros, R. Valentí, and F. Becca, Onedimensional spin liquid, collinear, and spiral phases from uncoupled chains to the triangular lattice, Phys. Rev. B 89, 235107 (2014).

[27] R. Kato et al. (unpublished).

[28] R. Kato, Y.-L. Liu, Y. Hosokoshi, S. Aonuma, and H. Sawa, Se-substitution and cation effects on the high-pressure molecular superconducior, $\beta-\mathrm{Me}_{4} \mathrm{~N}\left[\mathrm{Pd}(\mathrm{dmit})_{2}\right]_{2}-\mathrm{A}$ unique two-band system, Mol. Cryst. Liq. Cryst. 296, 217 (1997).

[29] K. Nakamura, Y. Yoshimoto, and M. Imada, Ab initio two-dimensional multiband low-energy models of $\mathrm{EtMe}_{3} \mathrm{Sb}\left[\mathrm{Pd}(\mathrm{dmit})_{2}\right]_{2}$ and $\kappa$-(BEDT-TTF $)_{2} \mathrm{Cu}(\mathrm{NCS})_{2}$ with comparisons to single-band models, Phys. Rev. B 86, 205117 (2012).

[30] K. Ueda, T. Tsumuraya, and R. Kato, Temperature dependence of crystal structures and band parameters in quantum spin liquid $\beta^{\prime}-\mathrm{Et}_{3} \mathrm{Sb}\left[\mathrm{Pd}(\mathrm{dmit})_{2}\right]_{2}$ and related materials, Crystals 8, 138 (2018).

[31] F. Aryasetiawan, M. Imada, A. Georges, G. Kotliar, S. Biermann, and A. I. Lichtenstein, Frequency-dependent local interactions and low-energy effective models from electronic structure calculations, Phys. Rev. B 70, 195104 (2004).

[32] M. Imada and T. Miyake, Electronic structure calculation by first principles for strongly correlated electron systems, J. Phys. Soc. Jpn. 79, 112001 (2010).

[33] T. Koretsune, Y. Motome, and A. Furusaki, Exact diagonalization study of Mott transition in the Hubbard model on an anisotropic triangular lattice, J. Phys. Soc. Jpn. 76, 074719 (2007).

[34] P. Hohenberg and W. Kohn, Inhomogeneous electron gas, Phys. Rev. 136, B864 (1964).

[35] W. Kohn and L. J. Sham, Self-Consistent Equations Including Exchange and Correlation Effects, Phys. Rev. 140, A1133 (1965).

[36] D. R. Hamann, Optimized norm-conserving Vanderbilt pseudopotentials, Phys. Rev. B 88, 085117 (2013). 
[37] M. Schlipf and F. Gygi, Optimization algorithm for the generation of ONCV pseudopotentials, Comput. Phys. Commun. 196, 36 (2015).

[38] P. Giannozzi, O. Andreussi, T. Brumme, O. Bunau, M. B. Nardelli, M. Calandra, R. Car, C. Cavazzoni, D. Ceresoli, M. Cococcioni et al., Advanced capabilities for materials modelling with Quantum ESPRESSO, J. Phys.: Condens. Matter 29, 465901 (2017).

[39] J. P. Perdew, K. Burke, and M. Ernzerhof, Generalized Gradient Approximation Made Simple, Phys. Rev. Lett. 77, 3865 (1996).

[40] K. Nakamura, Y. Yoshimoto, Y. Nomura, T. Tadano, M. Kawamura, T. Kosugi, K. Yoshimi, T. Misawa, and Y. Motoyama, RESPACK: An ab initio tool for derivation of effective low-energy model of material, Comput. Phys. Commun. 261, 107781 (2021)

[41] H. Kino and H. Fukuyama, Phase diagram of two-dimensional organic conductors: (BEDT-TTF) ${ }_{2} X$, J. Phys. Soc. Jpn. 65, 2158 (1996).

[42] K. Momma and F. Izumi, Vesta 3 for three-dimensional visualization of crystal, volumetric and morphology data, J. Appl. Crystallogr. 44, 1272 (2011).

[43] K. Nakamura, Y. Yoshimoto, Y. Nohara, and M. Imada, Ab initio low-dimensional physics opened up by dimensional downfolding: application to LaFeAsO, J. Phys. Soc. Jpn. 79, 123708 (2010).
[44] A. V. Knyazev, Toward the optimal preconditioned eigensolver: Locally optimal block preconditioned conjugate gradient method, SIAM J. Sci. Comput. 23, 517 (2001).

[45] M. Kawamura, K. Yoshimi, T. Misawa, Y. Yamaji, S. Todo, and N. Kawashima, Quantum lattice model solver $\mathcal{H} \Phi$, Comput. Phys. Commun. 217, 180 (2017).

[46] E. P. Kenny, G. David, N. Ferré, A. C. Jacko, and B. J. Powell, Frustration, ring exchange, and the absence of long-range order in $\mathrm{EtMe}_{3} \mathrm{Sb}\left[\mathrm{Pd}(\mathrm{dmit})_{2}\right]_{2}$ : From first principles to many-body theory, Phys. Rev. Mater. 4, 044403 (2020).

[47] A. Pustogow, M. Bories, A. Löhle, R. Rösslhuber, E. Zhukova, B. Gorshunov, S. Tomić, J. A. Schlueter, R. Hübner, T. Hiramatsu et al., Quantum spin liquids unveil the genuine Mott state, Nat. Mater. 17, 773 (2018).

[48] S. Yunoki and S. Sorella, Two spin liquid phases in the spatially anisotropic triangular Heisenberg model, Phys. Rev. B 74, 014408 (2006).

[49] E. P. Kenny, A. C. Jacko, and B. J. Powell, $x-\left[\operatorname{Pd}(\mathrm{dmit})_{2}\right]_{2}$ as a quasi-one-dimensional scalene Heisenberg model, Phys. Rev. Mater. 5, 084412 (2021).

[50] K. Ido, K. Yoshimi, T. Misawa, and M. Imada (unpublished).

[51] S. Fujiyama and R. Kato, Fragmented Electronic Spins with Quantum Fluctuations in Organic Mott Insulators Near a Quantum Spin Liquid, Phys. Rev. Lett. 122, 147204 (2019). 